\title{
Fully summing mappings between Banach spaces
}

\author{
by \\ Mário C. Matos (Campinas) and \\ Daniel M. Pellegrino (João Pessoa)
}

\begin{abstract}
We introduce and investigate the non- $n$-linear concept of fully summing mappings; if $n=1$ this concept coincides with the notion of nonlinear absolutely summing mappings and in this sense this article unifies these two theories. We also introduce a non- $n$-linear definition of Hilbert-Schmidt mappings and sketch connections between this concept and fully summing mappings.
\end{abstract}

1. Introduction. In the last years, several polynomial and multilinear generalizations of the concept of absolutely summing operators between Banach spaces have been exhaustively investigated and several nice results have appeared. Recently, a completely nonlinear approach to absolute summability was introduced by Matos [4]; the notion of absolutely summing mappings proposed in [4] generalizes, to arbitrary mappings, the concepts of absolutely summing operators and polynomials. If we consider an $n$-linear mapping from $E_{1} \times \cdots \times E_{n}$ into $F$ as a map (of one variable) from the Banach space $E=E_{1} \times \cdots \times E_{n}$ to $F$, then [4] also generalizes the notion of absolutely summing $n$-linear mappings. In some sense, in [4] we have a unified concept for arbitrary absolutely summing mappings (in one variable).

In this paper we revisit [4], introducing a general concept of fully summing mappings of several variables and, by using a unified treatment, we extend the results of [4] to arbitrary mappings of several variables. Restricting our arguments to the case $n=1$, we recover the results from [4]. We also extend the concept of Hilbert-Schmidt operators to arbitrary mappings and sketch connections between absolutely summing and Hilbert-Schmidt mappings.

2000 Mathematics Subject Classification: 46T99, 46G20.

Key words and phrases: Banach spaces, holomorphic mappings, absolutely summing, Hilbert-Schmidt mappings.

The second named author is partially supported by Instituto do Milênio IM-AGIMB, CNPq and Fundação de Apoio à Pesquisa do Estado da Paraíba-FAPESQ. 
2. Background and notation. Throughout this paper $\mathbb{N}$ denotes the set of all positive integers, and $E, E_{1}, \ldots, E_{n}, F$ represent Banach spaces over $\mathbb{K}$, where $\mathbb{K}$ represents the field of all scalars (complex or real). Given a natural number $n \geq 2$, the Banach space of all continuous $n$-linear mappings from $E_{1} \times \cdots \times E_{n}$ into $F$ endowed with the sup norm will be denoted by $\mathcal{L}\left(E_{1}, \ldots, E_{n} ; F\right)$. For $p>0$, the vector space of all sequences $\left(x_{j}\right)_{j=1}^{\infty}$ in $E$ such that $\left\|\left(x_{j}\right)_{j=1}^{\infty}\right\|_{p}=\left(\sum_{j=1}^{\infty}\left\|x_{j}\right\|^{p}\right)^{1 / p}<\infty$ is denoted by $l_{p}(E)$, and $l_{p}^{w}(E)$ represents the linear space of the sequences $\left(x_{j}\right)_{j=1}^{\infty}$ in $E$ such that

$$
\left(\varphi\left(x_{j}\right)\right)_{j=1}^{\infty} \in l_{p}
$$

for every continuous linear functional $\varphi: E \rightarrow \mathbb{K}$. In $l_{p}^{w}(E)$ we consider $\|\cdot\|_{w, p}$ given by

$$
\left\|\left(x_{j}\right)_{j=1}^{\infty}\right\|_{w, p}=\sup _{\varphi \in B_{E^{\prime}}}\left\|\left(\varphi\left(x_{j}\right)\right)_{j=1}^{\infty}\right\|_{p} .
$$

We also define $l_{p}^{u}(E)$ by

$$
l_{p}^{u}(E)=\left\{\left(x_{j}\right)_{j=1}^{\infty} \in l_{p}^{w}(E) ; \lim _{k \rightarrow \infty}\left\|\left(x_{j}\right)_{j=k}^{\infty}\right\|_{w, p}=0\right\} .
$$

Let us recall some fruitful nonlinear concepts related to absolute summability:

- An $n$-homogeneous polynomial $P: E \rightarrow F$ is called absolutely $(p ; q)$ summing (or $(p ; q)$-summing) if $\left(P\left(x_{j}\right)\right)_{j=1}^{\infty} \in l_{p}(F)$ for all $\left(x_{j}\right)_{j=1}^{\infty}$ $\in l_{q}^{u}(E)$. Analogously, an $n$-linear mapping $T: E_{1} \times \cdots \times E_{n} \rightarrow$ $F$ is absolutely $\left(p ; q_{1}, \ldots, q_{n}\right)$-summing (or $\left(p ; q_{1}, \ldots, q_{n}\right)$-summing) if $\left(T\left(x_{j}^{(1)}, \ldots, x_{j}^{(n)}\right)\right)_{j=1}^{\infty} \in l_{p}(F)$ for all $\left(x_{j}^{(k)}\right)_{j=1}^{\infty} \in l_{q_{k}}^{u}\left(E_{k}\right), k=1, \ldots, n$.

- If $A \subset E$ is an open set, an arbitrary mapping $f: A \rightarrow F$ is said to be absolutely $(p ; q)$-summing at $a \in A$ if $\left(f\left(a+x_{j}\right)-f(a)\right)_{j=1}^{\infty} \in l_{p}(F)$ whenever $\left(x_{j}\right)_{j=1}^{\infty} \in l_{q}^{u}(E)$ and $a+x_{j} \in A$ for each $j \in \mathbb{N}$.

- An $n$-linear mapping $T: E_{1} \times \cdots \times E_{n} \rightarrow F$ is fully $\left(p ; q_{1}, \ldots, q_{n}\right)$ summing if

$$
\left(T\left(x_{j_{1}}^{(1)}, \ldots, x_{j_{n}}^{(n)}\right)\right)_{j_{1}, \ldots, j_{n}=1}^{\infty} \in l_{p}(F)
$$

for all $\left(x_{j}^{(k)}\right)_{j=1}^{\infty} \in l_{q_{k}}^{u}\left(E_{k}\right), k=1, \ldots, n$.

The concept of absolutely summing mappings for scalar-valued mappings is due to Pietsch, and the general case was first investigated by Alencar and Matos in [1]. The general definition of absolutely summing mappings is due to Matos [4] (see also [3] and [6]). The special case of fully summing multilinear mappings was introduced by Matos in [5] and independently by Bombal, Pérez-García and Villanueva [2] with another name ("multiple summing mappings"). The class of fully summing mappings enjoys several nice properties such as Grothendieck's theorem, coincidence results, inclusion results, etc. (see [5], [7]-[10]). 
Next, we introduce the concept of "fully summing" for arbitrary mappings of several variables and we obtain examples and characterizations of arbitrary fully summing mappings.

Definition 1. Let $E_{1}, \ldots, E_{n}$ and $F$ be Banach spaces over $\mathbb{K}, p, q_{j} \geq 1$, $j=1, \ldots, n$, and $A \subset E_{1} \times \cdots \times E_{n}$ be an open set. A map $f: A \rightarrow F$ is fully $\left(p ; q_{1}, \ldots, q_{n}\right)$-summing at $a \in A$ if there exist $\delta_{1}, \ldots, \delta_{n}>0$ with

$$
B_{\delta_{1}}\left(a_{1}\right) \times \cdots \times B_{\delta_{n}}\left(a_{n}\right) \subset A
$$

such that for every $\left(x_{j}^{(k)}\right)_{j=1}^{\infty} \in l_{q_{k}}^{u}\left(E_{k}\right)$ with $\left\|x_{j}^{(k)}\right\| \leq \delta_{k}, j \in \mathbb{N}, k=1, \ldots, n$, we have

$$
\left(f\left(a+\left(x_{j_{1}}^{(1)}, \ldots, x_{j_{n}}^{(n)}\right)\right)-f(a)\right)_{j_{1}, \ldots, j_{n}=1}^{\infty} \in l_{p}(F) .
$$

In this case we write $f \in \mathcal{F}_{\mathrm{fs}(a)\left(p ; q_{1}, \ldots, q_{n}\right)}(A ; F)$.

The concept of "fully summing at a given point" is a local property and there is no loss of generality if we deal with the case $A=E_{1} \times \cdots \times E_{n}$, since the values of $f$ at points outside $A$ are irrelevant.

If $f$ is $n$-linear, $A=E_{1} \times \cdots \times E_{n}$ and $a=0$, we write $\mathcal{L}_{\mathrm{fs}\left(p ; q_{1}, \ldots, q_{n}\right)}$ instead of $\mathcal{F}_{\mathrm{fs}}(a)\left(p ; q_{1}, \ldots, q_{n}\right)$, since in this case we have the well known concept of fully (multiple) $\left(p ; q_{1}, \ldots, q_{n}\right)$-summing mappings. For multilinear mappings, it is not hard to prove that if $T$ is fully $\left(p ; q_{1}, \ldots, q_{n}\right)$-summing at $a \neq 0$, then $T=0$. Our first example shows that this is no longer valid if $T$ is not $n$-linear:

ExAmple 1. Let $E$ be an infinite-dimensional Banach space and choose $0 \neq e_{1}, e_{2} \in E$. Consider $f: E \times E \rightarrow \mathbb{K}$ defined by $f(x, y)=1$ except when there are scalars $\lambda_{1}, \lambda_{2}$ so that $x=e_{1}+\lambda_{1} e_{2}$ and $y=e_{1}+\lambda_{2} e_{2}$; in this case $f(x, y)=1+\lambda_{1} \lambda_{2}$. If $\left(x_{j}\right)_{j=1}^{\infty},\left(y_{j}\right)_{j=1}^{\infty} \in l_{1}^{u}(E)$, let us estimate

$$
\sum_{j, k=1}^{\infty}\left|f\left(e_{1}+x_{j}, e_{1}+y_{k}\right)-f\left(e_{1}, e_{1}\right)\right| .
$$

Note that we only have contributions to the sum when $x_{j}$ and $y_{k}$ belong to $G:=\left\langle e_{2}\right\rangle$. So, there is no loss of generality in supposing $\left(x_{j}\right)_{j=1}^{\infty},\left(y_{j}\right)_{j=1}^{\infty} \in$ $l_{1}^{u}(G)$. Since $G$ is finite-dimensional, we have $\left(x_{j}\right)_{j=1}^{\infty},\left(y_{j}\right)_{j=1}^{\infty} \in l_{1}(G)$ with $x_{j}=\lambda_{j} e_{2}$ and $y_{k}=\eta_{k} e_{2}$, and

$$
\begin{aligned}
\sum_{j, k=1}^{\infty}\left|f\left(e_{1}+x_{j}, e_{1}+y_{k}\right)-f\left(e_{1}, e_{1}\right)\right| & =\sum_{j, k=1}^{\infty}\left|1+\lambda_{j} \eta_{k}-1\right| \\
& =\sum_{j=1}^{\infty}\left|\lambda_{j}\right| \sum_{k=1}^{\infty}\left|\eta_{k}\right|<\infty .
\end{aligned}
$$

Hence $f$ is fully $(1 ; 1,1)$-summing at $\left(e_{1}, e_{1}\right)$. 
The next example shows that for maps of several variables, the concept of fully summing mappings is interesting only in the nonlinear case, since for linear maps we have a special behavior:

EXAMPLE 2. Let $p, q_{j} \geq 1$ and $n>1$ a natural number. If $T: E_{1} \times \cdots \times$ $E_{n} \rightarrow \mathbb{K}$ is linear (nonnull) in $E=E_{1} \times \cdots \times E_{n}$, then $T$ fails to be fully $\left(p ; q_{1}, \ldots, q_{n}\right)$-summing at the origin. In fact, there are $\varphi_{k} \in E_{k}^{\prime}$ so that

$$
T\left(x_{1}, \ldots, x_{n}\right)=\varphi_{1}\left(x_{1}\right)+\cdots+\varphi_{n}\left(x_{n}\right) .
$$

Since $T$ is nonnull, some of the $\varphi_{k}$ are nonnull. Suppose, for example, $\varphi_{1} \neq 0$. Choose $x \in E_{1}$ such that $\varphi_{1}(x) \neq 0$, and for every $0 \neq \lambda \in \mathbb{K}$, set

$$
\begin{aligned}
\left(x_{j}^{(1)}\right)_{j=1}^{\infty} & =(\lambda x, 0,0,0, \ldots), \\
\left(x_{j}^{(k)}\right)_{j=1}^{\infty} & =(0,0, \ldots) \quad \text { for every } k=2, \ldots, n .
\end{aligned}
$$

Hence

$$
\sum_{j_{1}, \ldots, j_{n}=1}^{\infty}\left|T\left(x_{j_{1}}^{(1)}, \ldots, x_{j_{n}}^{(n)}\right)\right|^{p}=\sum_{j_{2}, \ldots, j_{n}=1}^{\infty}\left|\varphi_{1}(\lambda x)\right|^{p}=\infty .
$$

ExAmple 3 . If $f\left(x_{1}, \ldots, x_{n}\right)=f_{1}\left(x_{1}\right) \cdots f_{n-1}\left(x_{n-1}\right) f_{n}\left(x_{n}\right)$ with $f_{j}$ : $E_{j} \rightarrow F_{j}$ absolutely $\left(p ; q_{j}\right)$-summing at zero, and $f_{j}(0)=0, j=1, \ldots, n$, with $F_{j}=\mathbb{K}, j=1, \ldots, n-1$, then $f$ is fully $\left(p ; q_{1}, \ldots, q_{n}\right)$-summing at zero.

3. Regular and fully regularly summing mappings. Absolutely summing operators, polynomials and multilinear mappings and also fully summing multilinear mappings are characterized by means of inequalities. In this section, adapting ideas from [4], we introduce some useful terminology that will be used in Section 4 to obtain characterizations of fully summing mappings by means of inequalities.

Definition 2. Let $E_{1}, \ldots, E_{n}$ and $F$ be Banach spaces over $\mathbb{K}$ and $A \subset E_{1} \times \cdots \times E_{n}$ be an open set. A map $f: A \rightarrow F$ is $\left(p ; q_{1}, \ldots, q_{n}\right)$-regular at $a=\left(a_{1}, \ldots, a_{n}\right) \in A$ if there are $r_{1}, \ldots, r_{n}>0$ and $M \geq 0$ such that

$$
B_{r_{1}}\left(a_{1}\right) \times \cdots \times B_{r_{n}}\left(a_{n}\right) \subset A
$$

and

$$
\left\|f\left(a+\left(x_{1}, \ldots, x_{n}\right)\right)-f(a)\right\|^{p} \leq M\left\|x_{1}\right\|^{q_{1}} \ldots\left\|x_{n}\right\|^{q_{n}}
$$

for every $x_{k} \in E_{k}$ with $\left\|x_{k}\right\| \leq r_{k}, k=1, \ldots, n$.

Note that regular mappings and fully summing mappings have natural connections with cotype, as shown in the example below:

Example 4 . If $E_{1}, \ldots, E_{n}$ and $F$ are Banach spaces, $E_{j}$ has cotype $q_{j}$ and $f$ is $\left(p ; q_{1}, \ldots, q_{n}\right)$-regular at $a$, then $f \in \mathcal{F}_{\mathrm{fs}(a)(p ; 1, \ldots, 1)}(A ; F)$. In fact, it 
suffices to observe that

$$
\sum_{j_{1}, \ldots, j_{n}=1}^{\infty}\left\|f\left(a+\left(x_{j_{1}}^{(1)}, \ldots, x_{j_{n}}^{(n)}\right)\right)-f(a)\right\|^{p} \leq M\left(\sum_{j=1}^{\infty}\left\|x_{j}^{(1)}\right\|^{q_{1}}\right) \cdots\left(\sum_{j=1}^{\infty}\left\|x_{j}^{(n)}\right\|^{q_{n}}\right)
$$

and recall that $\operatorname{id}_{E_{j}}$ is $\left(q_{j} ; 1\right)$-summing. One should not expect to obtain similar coincidences for regular mappings $f: E_{1} \times \cdots \times E_{n} \rightarrow F$ by exploring the cotype of $F$. For example, $f: c_{0} \rightarrow \mathbb{K}$ given by $f(x)=\|x\|$ is $(2 ; 2)$ regular at zero but is not $(2 ; 1)$-summing.

ExAMPLE 5. Let $f_{j}: A_{j} \rightarrow F_{j}$ be such that $0 \in A_{j} \subset E_{j}, A_{j}$ is open and each $f_{j}$ is absolutely $\left(p ; q_{j}\right)$-summing at zero, $f_{j}(0)=0, j=1, \ldots, n$. If $T: F_{1} \times \cdots \times F_{n} \rightarrow F$ is $(1 ; 1, \ldots, 1)$-regular at zero, then $T\left(f_{1}, \ldots, f_{n}\right)$ is fully $\left(p ; q_{1}, \ldots, q_{n}\right)$-summing at zero.

Definition 3. Let $E_{1}, \ldots, E_{n}$ and $F$ be Banach spaces and $A \subset E_{1} \times$ $\cdots \times E_{n}$ be an open set. A map $f: A \rightarrow F$ is fully regularly $\left(p ; q_{1}, \ldots, q_{n}\right)$ summing at $a=\left(a_{1}, \ldots, a_{n}\right) \in A$ if there exist $\delta_{1}, \ldots, \delta_{n}>0$ with

$$
B_{\delta_{1}}\left(a_{1}\right) \times \cdots \times B_{\delta_{n}}\left(a_{n}\right) \subset A
$$

such that for every $\left(x_{j}^{(k)}\right)_{j=1}^{\infty} \in l_{q_{k}}\left(E_{k}\right)$ with $\left\|x_{j}^{(k)}\right\| \leq \delta_{k}, j \in \mathbb{N}, k=1, \ldots, n$, we have

$$
\left(f\left(a+\left(x_{j_{1}}^{(1)}, \ldots, x_{j_{n}}^{(n)}\right)\right)-f(a)\right)_{j_{1}, \ldots, j_{n}=1}^{\infty} \in l_{p}(F) .
$$

In this case we write $f \in \mathcal{F}_{\text {frs }(a)\left(p ; q_{1}, \ldots, q_{n}\right)}(A ; F)$.

It is easy to prove that if $f$ is $\left(p ; q_{1}, \ldots, q_{n}\right)$-regular at $a$, then $f \in$ $\mathcal{F}_{\text {frs }(a)\left(p ; q_{1}, \ldots, q_{n}\right)}(A ; F)$. The converse also holds, but we need some preliminary results to prove this.

If $T$ is an $n$-linear mapping, multilinearity implies that $T\left(a_{1}, \ldots, a_{n}\right)=$ $T(0, \ldots, 0)=0$ if $a_{j}=0$ for some $j$, that is, the map is constant (zero) in some directions. For nonlinear mappings which are $\left(p ; q_{1}, \ldots, q_{n}\right)$-summing at $a=\left(a_{1}, \ldots, a_{n}\right)$ we have a similar behavior:

LEMMA 1. If $f$ is fully regularly $\left(p ; q_{1}, \ldots, q_{n}\right)$-summing at $\left(a_{1}, \ldots, a_{n}\right)$, then there are $\eta_{k}>0, k=1, \ldots, n$, such that

$$
\begin{aligned}
f\left(a_{1}+x_{1}, \ldots, a_{n-1}+x_{n-1}, a_{n}\right) & =f\left(a_{1}, a_{2}, \ldots, a_{n}\right), \\
\vdots & \\
f\left(a_{1}, a_{2}+x_{2}, \ldots, a_{n}+x_{n}\right) & =f\left(a_{1}, a_{2}, \ldots, a_{n}\right)
\end{aligned}
$$

whenever $\left\|x_{k}\right\| \leq \eta_{k}, k=1, \ldots, n$.

Proof. Suppose, for example, that there exist $x_{2}, \ldots, x_{n}$ sufficiently small so that

$$
f\left(a_{1}, a_{2}+x_{2}, \ldots, a_{n}+x_{n}\right) \neq f\left(a_{1}, a_{2}, \ldots, a_{n}\right) .
$$


By defining $\left(z_{j}^{(1)}\right)=(0,0,0, \ldots)$ in $E_{1}$ and $\left(z_{j}^{(r)}\right)=\left(x_{r}, 0,0,0, \ldots\right)$ in $E_{r}$, $r=2, \ldots, n$, we would obtain

$$
\begin{aligned}
& \sum_{j_{1}, \ldots, j_{n}=1}^{\infty}\left\|f\left(a_{1}+z_{j_{1}}^{(1)}, \ldots, a_{n}+z_{j_{n}}^{(n)}\right)-f\left(a_{1}, a_{2}, \ldots, a_{n}\right)\right\|^{p} \\
& \quad \geq \sum_{j_{1}=1}^{\infty}\left\|f\left(a_{1}, a_{2}+x_{2}, \ldots, a_{n}+x_{n}\right)-f\left(a_{1}, a_{2}, \ldots, a_{n}\right)\right\|^{p}=\infty .
\end{aligned}
$$

The next result extends [4, Theorem 2.5] to mappings of several variables.

THEOREM 1. The following assertions are equivalent for $f: A \rightarrow F$ with $A$ open in $E_{1} \times \cdots \times E_{n}$ and $a=\left(a_{1}, \ldots, a_{n}\right) \in A$ :

(a) $f$ is fully regularly $\left(p ; q_{1}, \ldots, q_{n}\right)$-summing at a.

(b) There exist $M, \delta>0$ such that

$$
\begin{aligned}
\left\|\left(f\left(a+\left(x_{j_{1}}^{(1)}, \ldots, x_{j_{n}}^{(n)}\right)\right)-f(a)\right)_{j_{1}, \ldots, j_{n}=1}^{\infty}\right\|_{p}^{p} & \\
& \leq M\left\|\left(x_{j}^{(1)}\right)_{j=1}^{\infty}\right\|_{q_{1}}^{q_{1}} \ldots\left\|\left(x_{j}^{(n)}\right)_{j=1}^{\infty}\right\|_{q_{n}}^{q_{n}}
\end{aligned}
$$

whenever $\left\|\left(x_{j}^{(k)}\right)_{j=1}^{\infty}\right\|_{q_{k}} \leq \delta, k=1, \ldots, n$.

(c) $f$ is $\left(p ; q_{1}, \ldots, q_{n}\right)$-regular at a.

Proof. (a) $\Rightarrow(\mathrm{b})$. Note that $f$ is fully regularly $\left(p ; q_{1}, \ldots, q_{n}\right)$-summing at $\left(a_{1}, \ldots, a_{n}\right)$ if and only if $g\left(x_{1}, \ldots, x_{n}\right):=f\left(a_{1}+x_{1}, \ldots, a_{n}+x_{n}\right)-$ $f\left(a_{1}, \ldots, a_{n}\right)$ is fully regularly $\left(p ; q_{1}, \ldots, q_{n}\right)$-summing at zero. So, for our purposes, it suffices to deal with the case $\left(a_{1}, \ldots, a_{n}\right)=(0, \ldots, 0)$ and $f(0, \ldots, 0)=0$.

From Lemma 1 , we can find $\eta_{1}, \ldots, \eta_{n}>0$ so that $\left\|x_{k}\right\| \leq \eta_{k}$ implies

$$
\begin{gathered}
f\left(0, x_{2}, \ldots, x_{n}\right)=0, \\
\vdots \\
f\left(x_{1}, \ldots, x_{n-1}, 0\right)=0 .
\end{gathered}
$$

If $m \in \mathbb{N}, X_{m}^{(k)} \in l_{q_{k}}\left(E_{k}\right)$ and $r_{m}^{(k)} \in \mathbb{N}, k=1, \ldots, n$, we define

$$
X_{m}^{(k)}=\left(x_{m, 1}^{(k)}, \ldots, x_{m, j}^{(k)}, \ldots\right)
$$

and

$$
X_{m}^{(k)}\left(r_{m}^{(k)}\right)=\left(x_{m, 1}^{(k)}, \ldots, x_{m, 1}^{(k)}, x_{m, 2}^{(k)}, \ldots, x_{m, 2}^{(k)}, \ldots\right),
$$

where each $x_{m, j}^{(k)}$ is repeated $r_{m}^{(k)}$ times.

Now, suppose that the result is false. Then, for every $m$, there are $X_{m}^{(k)}=$ $\left(x_{m, j}^{(k)}\right)_{j=1}^{\infty}$ so that

$$
\left\|X_{m}^{(k)}\right\|_{q_{k}}^{q_{k}}<\min \left\{\eta_{k}^{q_{k}}, m^{-(3 n+1)}\right\}
$$


and

$$
\sum_{\left(j_{1} \ldots, j_{n}\right) \in \mathbb{N}^{n}}\left\|f\left(x_{m, j_{1}}^{(1)}, \ldots, x_{m, j_{n}}^{(n)}\right)\right\|^{p}>m^{n+1}\left\|X_{m}^{(1)}\right\|_{q_{1}}^{q_{1}} \cdots\left\|X_{m}^{(n)}\right\|_{q_{n}}^{q_{n}}
$$

Note that $\left\|X_{m}^{(k)}\right\|_{q_{k}}^{q_{k}} \neq 0$ for every $k$ and $m$, because

$$
\left\|X_{m}^{(k)}\right\|_{q_{k}}^{q_{k}}<\eta_{k}^{q_{k}} \Rightarrow\left\|x_{m, j}^{(k)}\right\|^{q_{k}}<\eta_{k}^{q_{k}} \Rightarrow\left\|x_{m, j}^{(k)}\right\|<\eta_{k} .
$$

In fact, if $\left\|X_{m}^{(k)}\right\|_{q_{k}}^{q_{k}}=0$ for some $k,(3.3)$ would imply $f\left(x_{m, j_{1}}^{(1)}, \ldots, x_{m, j_{n}}^{(n)}\right)=0$ and from $(3.2)$ we would have $0>0$.

Define $\varepsilon=1 / n$ and

$$
r_{m}^{(k)}=\left[\frac{1}{m^{2+\varepsilon}\left\|X_{m}^{(k)}\right\|_{q_{k}}^{q_{k}}}\right]:=\sup \left\{r \in \mathbb{N} ; r \leq \frac{1}{m^{2+\varepsilon}\left\|X_{m}^{(k)}\right\|_{q_{k}}^{q_{k}}}\right\} .
$$

It is worth remarking that, for every $m$ and $k$, the number $r_{m}^{(k)}$ is in fact greater than or equal to 1 , because (3.1) implies that

$$
\frac{1}{m^{2+\varepsilon}\left\|X_{m}^{(k)}\right\|_{q_{k}}^{q_{k}}}>m^{3 n+1-2-\varepsilon} \geq 1
$$

Note that for our choice of $r_{m}^{(k)}$ we have

$$
\begin{aligned}
\sum_{m \in \mathbb{N}}\left\|X_{m}^{(k)}\left(m r_{m}^{(k)}\right)\right\|_{q_{k}}^{q_{k}} & =\sum_{m \in \mathbb{N}} m r_{m}^{(k)}\left\|X_{m}^{(k)}\right\|_{q_{k}}^{q_{k}} \\
& \leq \sum_{m \in \mathbb{N}} m \frac{1}{m^{2+\varepsilon}\left\|X_{m}^{(k)}\right\|_{q_{k}}^{q_{k}}}\left\|X_{m}^{(k)}\right\|_{q_{k}}^{q_{k}}=\sum_{m \in \mathbb{N}} \frac{1}{m^{1+\varepsilon}}<\infty
\end{aligned}
$$

and, since $f$ is fully regularly $\left(p ; q_{1}, \ldots, q_{n}\right)$-summing, we obtain

$$
\sum_{\left(m_{1}, \ldots, m_{n}, j_{1}, \ldots, j_{n}\right) \in \mathbb{N}^{2 n}} m_{1} \cdots m_{n} r_{m_{1}}^{(1)} \cdots r_{m_{n}}^{(n)}\left\|f\left(x_{m_{1}, j_{1}}^{(1)}, \ldots, x_{m_{n}, j_{n}}^{(n)}\right)\right\|^{p}<\infty .
$$

Now, call on (3.2) and (3.5) to obtain

$$
\begin{aligned}
& \sum_{m \in \mathbb{N}} m^{2 n+1} r_{m}^{(1)} \cdots r_{m}^{(n)}\left\|X_{m}^{(1)}\right\|_{q_{1}}^{q_{1}} \cdots\left\|X_{m}^{(n)}\right\|_{q_{n}}^{q_{n}} \\
& =\sum_{m \in \mathbb{N}} m^{n} m^{n+1} r_{m}^{(1)} \cdots r_{m}^{(n)}\left\|X_{m}^{(1)}\right\|_{q_{1}}^{q_{1}} \cdots\left\|X_{m}^{(n)}\right\|_{q_{n}}^{q_{n}} \\
& \quad \stackrel{(3.2)}{\leq} \sum_{m \in \mathbb{N}} m^{n} r_{m}^{(1)} \cdots r_{m}^{(n)} \sum_{\left(j_{1} \ldots, j_{n}\right) \in \mathbb{N}^{n}} \| f\left(x_{m, j_{1}}^{(1)}, \ldots, x_{m, j_{n}}^{(n)} \|^{p} \stackrel{(3.5)}{<} \infty .\right.
\end{aligned}
$$

On the other hand, for every $k=1, \ldots, n$ and every $m \in \mathbb{N}$,

$$
\frac{1}{m^{2+\varepsilon}\left\|X_{m}^{(k)}\right\|_{q_{k}}^{q_{k}}}-1 \leq r_{m}^{(k)} \leq \frac{1}{m^{2+\varepsilon}\left\|X_{m}^{(k)}\right\|_{q_{k}}^{q_{k}}}
$$


and multiplying (3.7) term by term, we have

$$
\frac{\prod_{k=1}^{n}\left(1-m^{2+\varepsilon}\left\|X_{m}^{(k)}\right\|_{q_{k}}^{q_{k}}\right)}{m^{(2+\varepsilon) n}\left\|X_{m}^{(1)}\right\|_{q_{1}}^{q_{1}} \cdots\left\|X_{m}^{(n)}\right\|_{q_{n}}^{q_{n}}} \leq \prod_{k=1}^{n} r_{m}^{(k)} \leq \frac{1}{m^{(2+\varepsilon) n}\left\|X_{m}^{(1)}\right\|_{q_{1}}^{q_{1}} \ldots\left\|X_{m}^{(n)}\right\|_{q_{n}}^{q_{n}}}
$$

and so

$$
\prod_{k=1}^{n}\left(1-m^{2+\varepsilon}\left\|X_{m}^{(k)}\right\|_{q_{k}}^{q_{k}}\right) \leq m^{(2+\varepsilon) n} \prod_{k=1}^{n}\left(r_{m}^{(k)}\left\|X_{m}^{(k)}\right\|_{q_{k}}^{q_{k}}\right) \leq 1 .
$$

From (3.1) we obtain, for every $k=1, \ldots, n$,

$$
\lim _{m \rightarrow \infty} m^{2+\varepsilon}\left\|X_{m}^{(k)}\right\|_{q_{k}}^{q_{k}} \leq \lim _{m \rightarrow \infty} m^{2+\varepsilon} m^{-(3 n+1)}=\lim _{m \rightarrow \infty} m^{2+1 / n-3 n-1}=0 .
$$

From (3.8) and (3.9), it follows that

$$
\lim _{m \rightarrow \infty} r_{m}^{(1)} \cdots r_{m}^{(n)} m^{(2+\varepsilon) n}\left\|X_{m}^{(1)}\right\|_{q_{1}}^{q_{1}} \cdots\left\|X_{m}^{(n)}\right\|_{q_{n}}^{q_{n}}=1
$$

that is,

$$
\lim _{m \rightarrow \infty} r_{m}^{(1)} \cdots r_{m}^{(n)} m^{2 n+1}\left\|X_{m}^{(1)}\right\|_{q_{1}}^{q_{1}} \cdots\left\|X_{m}^{(n)}\right\|_{q_{n}}^{q_{n}}=1
$$

and (3.10) contradicts the convergence of the series in (3.6).

(b) $\Rightarrow$ (c). If $x_{k} \in E_{k}, k=1, \ldots, n$, satisfy $\left\|x_{k}\right\|<\delta$, then consider

$$
\left(x_{j}^{(k)}\right)_{j=1}^{\infty}=\left(x_{k}, 0,0, \ldots\right) \quad \text { for every } k=1, \ldots, n .
$$

Hence

$$
\begin{aligned}
\left\|f\left(a+\left(x_{1}, \ldots, x_{n}\right)\right)-f(a)\right\|^{p} & \leq\left\|\left(f\left(a+\left(x_{j_{1}}^{(1)}, \ldots, x_{j_{n}}^{(n)}\right)\right)-f(a)\right)_{j_{1}, \ldots, j_{n}=1}^{\infty}\right\|_{p}^{p} \\
& \leq M\left\|\left(x_{j}^{(1)}\right)_{j=1}^{\infty}\right\|_{q_{1}}^{q_{1}} \cdots\left\|\left(x_{j}^{(n)}\right)_{j=1}^{\infty}\right\|_{q_{n}}^{q_{n}} \\
& =M\left\|x_{1}\right\|^{q_{1}} \cdots\left\|x_{n}\right\|^{q_{n}}
\end{aligned}
$$

when $\left\|x_{k}\right\|<\delta, k=1, \ldots, n$.

(c) $\Rightarrow(\mathrm{a})$. If $f$ is $\left(p ; q_{1}, \ldots, q_{n}\right)$-regular at $a$, then

$$
\left\|f\left(a+\left(x_{1}, \ldots, x_{n}\right)\right)-f(a)\right\|^{p} \leq M\left\|x_{1}\right\|^{q_{1}} \cdots\left\|x_{n}\right\|^{q_{n}}
$$

if $\left\|x_{k}\right\|<r_{k}, k=1, \ldots, n$. Hence, if $\left\|x_{j}^{(k)}\right\|<r_{k}, j \in \mathbb{N}, k=1, \ldots, n$, from (3.11) we obtain

$$
\sum_{j_{1}, \ldots, j_{n}=1}^{\infty}\left\|f\left(a+\left(x_{j_{1}}^{(1)}, \ldots, x_{j_{n}}^{(n)}\right)\right)-f(a)\right\|^{p} \leq \sum_{j_{1}, \ldots, j_{n}=1}^{\infty} M\left\|x_{j_{1}}^{(1)}\right\|^{q_{1}} \cdots\left\|x_{j_{n}}^{(n)}\right\|^{q_{n}}
$$

and so $f$ is fully regularly $\left(p ; q_{1}, \ldots, q_{n}\right)$-summing at $a$.

\section{Characterization of fully summing mappings by inequalities.}

If $f: A \rightarrow F$ is fully $\left(p ; q_{1}, \ldots, q_{n}\right)$-summing at $a=\left(a_{1}, \ldots, a_{n}\right) \in A \subset$ $E_{1} \times \cdots \times E_{n}$, let $\delta$ be so that $B_{\delta}\left(a_{1}\right) \times \cdots \times B_{\delta}\left(a_{n}\right) \subset A$ and 


$$
\begin{aligned}
\left(x_{j}^{(k)}\right)_{j=1}^{\infty} \in l_{q_{k}}^{u}\left(E_{k}\right) & \text { with }\left\|x_{j}^{(k)}\right\|<\delta \\
& \Rightarrow\left(f\left(a+\left(x_{j_{1}}^{(1)}, \ldots, x_{j_{n}}^{(n)}\right)\right)-f(a)\right)_{j_{1}, \ldots, j_{n}=1}^{\infty} \in l_{p}(F) .
\end{aligned}
$$

We can consider the natural associated mapping

$$
\Psi_{f}: V_{q_{1}, \delta}\left(E_{1}\right) \times \cdots \times V_{q_{n}, \delta}\left(E_{n}\right) \rightarrow l_{p}(F)
$$

given by

$$
\Psi_{f}\left(\left(x_{j}^{(1)}\right)_{j=1}^{\infty}, \ldots,\left(x_{j}^{(n)}\right)_{j=1}^{\infty}\right)=\left(f\left(a+\left(x_{j_{1}}^{(1)}, \ldots, x_{j_{n}}^{(n)}\right)\right)-f(a)\right)_{j_{1}, \ldots, j_{n}=1}^{\infty},
$$

where

$$
V_{q_{k}, \delta}\left(E_{k}\right)=\left\{\left(x_{j}^{(k)}\right)_{j=1}^{\infty} \in l_{q_{k}}^{u}\left(E_{k}\right) ;\left\|x_{j}^{(k)}\right\|<\delta \text { for every } j\right\}, k=1, \ldots, n .
$$

The next theorem shows nice useful connections between $\Psi_{f}$ and $f$, which will help us to characterize fully summing mappings.

Theorem 2. If $A \subset E_{1} \times \cdots \times E_{n}$ is an open set and $f: A \rightarrow F$ is fully $\left(p ; q_{1}, \ldots, q_{n}\right)$-summing at $a=\left(a_{1}, \ldots, a_{n}\right) \in A$, then $\Psi_{f}$ is fully regularly $\left(p ; q_{1}, \ldots, q_{n}\right)$-summing at zero.

Proof. Let $\delta$ be such that $B_{\delta}\left(a_{1}\right) \times \cdots \times B_{\delta}\left(a_{n}\right) \subset A$ and

$$
\begin{aligned}
\left(x_{j}^{(l)}\right)_{j=1}^{\infty} \in l_{q_{l}}^{u}\left(E_{l}\right) & \text { with }\left\|x_{j}^{(l)}\right\|<\delta \\
\Rightarrow & \left(f\left(a+\left(x_{j_{1}}^{(1)}, \ldots, x_{j_{n}}^{(n)}\right)\right)-f(a)\right)_{j_{1}, \ldots, j_{n}=1}^{\infty} \in l_{p}(F) .
\end{aligned}
$$

If $\left(X_{j}^{(l)}\right)_{j=1}^{\infty} \in l_{q_{l}}\left(l_{q_{l}}^{u}\left(E_{l}\right)\right)$ with $X_{j}^{(l)}=\left(x_{j, k}^{(l)}\right)_{k=1}^{\infty}$ and $\left\|X_{j}^{(l)}\right\|<\delta, l=1, \ldots, n$, then

Hence

$$
\left(x_{j, k}^{(l)}\right)_{j, k=1}^{\infty} \in l_{q_{l}}^{u}\left(E_{l}\right) \quad \text { and } \quad\left\|x_{j, k}^{(l)}\right\|<\delta .
$$

$$
\begin{aligned}
& \sum_{j_{1}, \ldots, j_{n}=1}^{\infty}\left\|\Psi_{f}\left(X_{j_{1}}^{(1)}, \ldots, X_{j_{n}}^{(n)}\right)\right\|^{p} \\
& =\sum_{j_{1}, \ldots, j_{n}=1}^{\infty}\left(\sum_{k_{1}, \ldots, k_{n}=1}^{\infty}\left\|f\left(a+\left(x_{j_{1}, k_{1}}^{(1)}, \ldots, x_{j_{n}, k_{n}}^{(n)}\right)\right)-f(a)\right\|^{p}\right)<\infty
\end{aligned}
$$

Now we state the main result of the paper:

Theorem 3. For an open set $A \subset E_{1} \times \cdots \times E_{n}, f: A \rightarrow F$ and $a=\left(a_{1}, \ldots, a_{n}\right) \in A$, the following statements are equivalent:

(a) $f$ is fully $\left(p ; q_{1}, \ldots, q_{n}\right)$-summing at a.

(b) There exist $M, \delta>0$ such that $B_{\delta}\left(a_{1}\right) \times \cdots \times B_{\delta}\left(a_{n}\right) \subset A$ and

$$
\begin{aligned}
\left\|\left(f\left(a+\left(x_{j_{1}}^{(1)}, \ldots, x_{j_{n}}^{(n)}\right)\right)-f(a)\right)_{j_{1}, \ldots, j_{n}=1}^{\infty}\right\|_{p}^{p} & \\
& \leq M\left\|\left(x_{j}^{(1)}\right)_{j=1}^{\infty}\right\|_{w, q_{1}}^{q_{1}} \cdots\left\|\left(x_{j}^{(n)}\right)_{j=1}^{\infty}\right\|_{w, q_{n}}^{q_{n}}
\end{aligned}
$$

whenever $\left(x_{j}^{(k)}\right)_{j=1}^{\infty} \in l_{q_{k}}^{u}\left(E_{k}\right)$ and $\left\|\left(x_{j}^{(k)}\right)_{j=1}^{\infty}\right\|_{w, q_{k}} \leq \delta, k=1, \ldots, n$. 
Proof. (a) $\Rightarrow$ (b). From Theorem 2, we know that $\Psi_{f}$ is fully regularly $\left(p ; q_{1}, \ldots, q_{n}\right)$-summing at zero and, from Theorem $1, \Psi_{f}$ is $\left(p ; q_{1}, \ldots, q_{n}\right)$ regular at zero. Hence there exist $M, \delta_{k}>0, k=1, \ldots, n$, such that

$$
\left\|\Psi_{f}\left(X^{(1)}, \ldots, X^{(n)}\right)\right\|_{p}^{p} \leq M\left\|X^{(1)}\right\|_{w, q_{1}}^{q_{1}} \cdots\left\|X^{(n)}\right\|_{w, q_{n}}^{q_{n}}
$$

whenever $X^{(k)}=\left(x_{j}^{(k)}\right)_{j=1}^{\infty} \in l_{q_{k}}^{u}\left(E_{k}\right)$ and $\left\|X^{(k)}\right\|_{w, q_{k}} \leq \delta_{k}, k=1, \ldots, n$. Therefore

$$
\begin{aligned}
\left\|\left(f\left(a+\left(x_{j_{1}}^{(1)}, \ldots, x_{j_{n}}^{(n)}\right)\right)-f(a)\right)_{j_{1}, \ldots, j_{n}=1}^{\infty}\right\|_{p}^{p} & \\
& \leq M\left\|\left(x_{j}^{(1)}\right)_{j=1}^{\infty}\right\|_{w, q_{1}}^{q_{1}} \cdots\left\|\left(x_{j}^{(n)}\right)_{j=1}^{\infty}\right\|_{w, q_{n}}^{q_{n}}
\end{aligned}
$$

when $\left(x_{j}^{(k)}\right)_{j=1}^{\infty} \in l_{q_{k}}^{u}\left(E_{k}\right)$ and $\left\|\left(x_{j}^{(k)}\right)_{j=1}^{\infty}\right\|_{w, q_{k}} \leq \delta_{k}, k=1, \ldots, n$.

(b) $\Rightarrow(\mathrm{a})$. Let $\left(x_{j}^{(k)}\right)_{j=1}^{\infty} \in l_{q_{k}}^{u}\left(E_{k}\right)$ and $\left\|x_{j}^{(k)}\right\|<\delta$ for every $j$ and $k$. We want to prove that the sequence $\left(f\left(a+\left(x_{j_{1}}^{(1)}, \ldots, x_{j_{n}}^{(n)}\right)\right)-f(a)\right)_{j_{1}, \ldots, j_{n}=1}^{\infty}$ belongs to $l_{p}(F)$. The idea of the proof is to divide this sequence into a finite number of sequences and show that each one belongs to $l_{p}(F)$.

Since $\left(x_{j}^{(k)}\right)_{j=1}^{\infty} \in l_{q_{k}}^{u}\left(E_{k}\right)$, there is a $j_{0}$ such that

From (b) we have

$$
\left\|\left(x_{j}^{(k)}\right)_{j=j_{0}}^{\infty}\right\|_{w, q_{k}} \leq \delta, \quad k=1, \ldots, n .
$$

$$
\left(f\left(a+\left(x_{j_{1}}^{(1)}, \ldots, x_{j_{n}}^{(n)}\right)\right)-f(a)\right)_{j_{1}, \ldots, j_{n}=j_{0}}^{\infty} \in l_{p}(F) .
$$

It is also clear that

$$
\left(f\left(a+\left(x_{j_{1}}^{(1)}, \ldots, x_{j_{n}}^{(n)}\right)\right)-f(a)\right)_{j_{1}, \ldots, j_{n}=1}^{j_{0}} \in l_{p}(F)
$$

and so it suffices to note that considering the other cases as situations of the type "some $j_{k}$ fixed between 1 and $j_{0}$ and other $j_{k}$ varying from $j_{0}$ to infinity" we have a finite number of situations and the corresponding sequences also belong to $l_{p}(F)$. For example, in order to prove that

$$
\left(f\left(a+\left(x_{1}^{(1)}, x_{j_{2}}^{(2)}, \ldots, x_{j_{n}}^{(n)}\right)\right)-f(a)\right)_{j_{2}, \ldots, j_{n}=j_{0}}^{\infty} \in l_{p}(F)
$$

it suffices to consider $\left(y_{j}\right)_{j=1}^{\infty}=\left(x_{1}^{(1)}, 0,0, \ldots\right)$ and hence $\left\|\left(y_{j}\right)_{j=1}^{\infty}\right\|_{w, q_{1}} \leq \delta$ and (b) asserts that

$$
\begin{aligned}
\|\left(f \left(a+\left(y_{j_{1}}, x_{j_{2}}^{(2)}, \ldots,\right.\right.\right. & \left.\left.\left.x_{j_{n}}^{(n)}\right)\right)-f(a)\right)_{j_{1}=1, j_{2}, \ldots, j_{n}=j_{0}}^{\infty} \|_{p}^{p} \\
& \leq M\left\|x_{1}^{(1)}\right\|^{q_{1}}\left\|\left(x_{j}^{(2)}\right)_{j=1}^{\infty}\right\|_{w, q_{2}}^{q_{2}} \cdots\left\|\left(x_{j}^{(n)}\right)_{j=1}^{\infty}\right\|_{w, q_{n}}^{q_{n}} .
\end{aligned}
$$

Since

$$
\begin{aligned}
\|\left(f \left(a+\left(x_{1}^{(1)},\right.\right.\right. & \left.\left.\left.x_{j_{2}}^{(2)}, \ldots, x_{j_{n}}^{(n)}\right)\right)-f(a)\right)_{j_{2}, \ldots, j_{n}=j_{0}}^{\infty} \|_{p}^{p} \\
& \leq\left\|\left(f\left(a+\left(y_{j_{1}}, x_{j_{2}}^{(2)}, \ldots, x_{j_{n}}^{(n)}\right)\right)-f(a)\right)_{j_{1}=1, j_{2}, \ldots, j_{n}=j_{0}}^{\infty}\right\|_{p}^{p},
\end{aligned}
$$

we obtain (4.2). The other cases are similar. 
5. Hilbert-Schmidt mappings. In the following, unless otherwise stated, $E$ and $F$ will denote Hilbert spaces and $A \subset E$ will be an open set.

In order to motivate our definition of a general Hilbert-Schmidt mapping from $E$ to $F$ at a point $a$, we recall the concept of Hilbert-Schmidt operator and make some remarks.

A linear operator from $E$ into $F$ is Hilbert-Schmidt if, for each orthonormal basis $\left(u_{i}\right)_{i \in I}$ of $E$, it follows that $\left(T\left(u_{i}\right)\right)_{i \in I} \in l_{2}(I ; F)$. Note that

$$
\sum_{j \in J}\left\|T\left(\lambda_{j} u_{j}\right)\right\|=\sum_{j \in J}\left|\lambda_{j}\right|\left\|T\left(u_{j}\right)\right\| \leq\left(\sum_{j \in J}\left|\lambda_{j}\right|^{2}\right)^{1 / 2}\left(\sum_{i \in J}\left\|T\left(u_{i}\right)\right\|^{2}\right)^{1 / 2}
$$

for all finite subsets $J$ of $I$ and $\lambda_{j} \in \mathbb{K}, j \in J$. In particular, $T$ is HilbertSchmidt if and only if there exist $\delta, M>0$ such that

$$
\sum_{j \in J}\left\|T\left(\lambda_{j} u_{j}\right)\right\| \leq M\left(\sum_{j \in J}\left|\lambda_{j}\right|^{2}\right)^{1 / 2}
$$

for all finite subsets $J$ of $I$ and $\left\|\left(\lambda_{j}\right)_{j \in J}\right\|_{2} \leq \delta$.

The above remarks give the motivation for the following concept.

Definition 4. A mapping $f$ defined in $A \subset E$ with values in $F$ is Hilbert-Schmidt at $a \in A$ if, for each orthonormal basis $\left(u_{i}\right)_{i \in I}$ of $E$, there are $M \geq 0$ and $\delta>0$ such that $B_{\delta}(a) \subset A$ and

$$
\sum_{j \in J}\left\|f\left(a+\lambda_{j} u_{j}\right)-f(a)\right\| \leq M\left\|\left(\lambda_{j}\right)_{j \in J}\right\|_{2}
$$

for all finite subsets $J$ of $I$ and $\lambda_{j} \in \mathbb{K}, j \in J$, with $\left\|\left(\lambda_{j}\right)_{j \in J}\right\|_{2} \leq \delta$.

REMARK 1. Note that, a priori, it is not natural to expect a constant $M$ which works for every orthonormal basis. In fact, if such an $M$ exists, considering $J=\{1\}$, we would have, for $0 \neq x \in E$ with $\|x\|<\delta$ (and considering $x /\|x\|$ as an element of an orthonormal basis),

$$
\|f(a+x)-f(a)\|=\left\|f\left(a+\|x\| \frac{x}{\|x\|}\right)-f(a)\right\| \leq M\left(\|x\|^{2}\right)^{1 / 2}=M\|x\|,
$$

and $f$ would necessarily be 1-regular at $a$.

EXAMPLE 6. If $k \in \mathbb{N}, k \geq 2$, then each continuous $k$-homogeneous polynomial $P$ from $E$ into $F$ is Hilbert-Schmidt at 0 . In fact, if $\left(u_{i}\right)_{i \in I}$ is an orthonormal basis of $E$, then

$$
\sum_{j \in J}\left\|P\left(\lambda_{j} u_{j}\right)\right\| \leq\|P\| \sum_{j \in J}\left|\lambda_{j}\right|^{k} \leq\|P\|\left\|\left(\lambda_{j}\right)_{j \in J}\right\|_{2}^{k} \leq\|P\|\left\|\left(\lambda_{j}\right)_{j \in J}\right\|_{2}
$$

for all finite subsets $J$ of $I$ and $\lambda_{j} \in \mathbb{K}, j \in J$, with $\left\|\left(\lambda_{j}\right)_{j \in J}\right\|_{2} \leq 1$. 
5.1. Hilbert-Schmidt mappings vs absolutely 1-summing mappings

Proposition 1. If $f$ defined in $A \subset E$ with values in $F$ is absolutely 1-summing at $a \in A$, then $f$ is Hilbert-Schmidt at a.

Proof. By hypothesis there are $D \geq 0$ and $d>0$ such that $B_{d}(a) \subset A$ and

$$
\sum_{j \in J}\left\|f\left(a+x_{j}\right)-f(a)\right\| \leq D\left\|\left(x_{j}\right)_{j \in J}\right\|_{w, 1}
$$

for all finite sequences $\left(x_{j}\right)_{j \in J}$ with $\left\|\left(x_{j}\right)_{j \in J}\right\|_{w, 1} \leq d$. Since for each orthonormal basis $\left(u_{i}\right)_{i \in I}$ of $E$ we have

$$
\left\|\left(\lambda_{j} u_{j}\right)_{j \in J}\right\|_{w, 1}=\left\|\left(\lambda_{j}\right)_{j \in J}\right\|_{2},
$$

it follows that $f$ is Hilbert-Schmidt at $a$.

THEOREM 4. If $f$ is a $C^{2}$ mapping defined on $A \subset E$ with values in $F$, then $f$ is Hilbert-Schmidt at $a \in A$ if, and only if, $f$ is absolutely 1-summing at a.

Proof. In view of Proposition 1, it suffices to show that if $f$ is HilbertSchmidt at $a$, then $f$ is absolutely 1-summing at $a$.

So, suppose that $f$ is Hilbert-Schmidt at $a$. From Taylor's formula, we have

$$
\left\|f\left(a+\lambda_{j} u_{j}\right)-f(a)-d f(a)\left(\lambda_{j} u_{j}\right)-\widehat{d}^{2} f(a)\left(\lambda_{j} u_{j}\right)\right\| \leq\left\|\lambda_{j} u_{j}\right\|^{2}
$$

if $\left\|\lambda_{j} u_{j}\right\|<\varepsilon$. So, if $\left\|\lambda_{j} u_{j}\right\|<\varepsilon$, we obtain

$$
\left\|d f(a)\left(\lambda_{j} u_{j}\right)\right\| \leq\left\|f\left(a+\lambda_{j} u_{j}\right)-f(a)\right\|+\left\|\widehat{d^{2}} f(a)\left(\lambda_{j} u_{j}\right)\right\|+\left\|\lambda_{j} u_{j}\right\|^{2} .
$$

Since $f$ is Hilbert-Schmidt at $a$ and $\widehat{d^{2}} f(a)$ is a 2-homogeneous polynomial (and hence Hilbert-Schmidt at zero), we can find positive constants $M, N, \delta$ such that

$$
\begin{aligned}
\sum_{j \in J} & \left\|d f(a)\left(\lambda_{j} u_{j}\right)\right\| \\
& \leq \sum_{j \in J}\left\|f\left(a+\lambda_{j} u_{j}\right)-f(a)\right\|+\sum_{j \in J}\left\|\widehat{d^{2}} f(a)\left(\lambda_{j} u_{j}\right)\right\|+\sum_{j \in J}\left\|\lambda_{j} u_{j}\right\|^{2} \\
& \leq M\left\|\left(\lambda_{j}\right)_{j \in J}\right\|_{2}+N\left\|\left(\lambda_{j}\right)_{j \in J}\right\|_{2}+\left\|\left(\lambda_{j}\right)_{j \in J}\right\|_{2} \\
& =(M+N+1)\left\|\left(\lambda_{j}\right)_{j \in J}\right\|_{2}
\end{aligned}
$$

if $\left\|\left(\lambda_{j}\right)_{j \in J}\right\|_{2}<\delta$.

Hence $d f(a)$ is Hilbert-Schmidt and thus absolutely 1-summing (since it is a linear mapping). Now, by invoking Taylor's formula again, we can conclude that $f$ is absolutely 1 -summing. In fact, Taylor's formula says that there exists $\varepsilon>0$ such that 


$$
\|f(a+x)-f(a)\| \leq\|d f(a)(x)\|+\left\|\widehat{d^{2}} f(a)(x)\right\|+\|x\|^{2}
$$

for every $x \in E$ with $\|x\|<\varepsilon$.

If $\left(x_{j}\right)_{j=1}^{\infty} \in l_{1}^{u}(E)$, choose $j_{0}$ so that $j \geq j_{0} \Rightarrow\left\|x_{j}\right\|<\varepsilon$. Hence

$$
\sum_{j=j_{0}}^{\infty}\left\|f\left(a+x_{j}\right)-f(a)\right\| \leq \sum_{j=j_{0}}^{\infty}\left\|d f(a)\left(x_{j}\right)\right\|+\sum_{j=j_{0}}^{\infty}\left\|\widehat{d^{2}} f(a)\left(x_{j}\right)\right\|+\sum_{j=j_{0}}^{\infty}\left\|x_{j}\right\|^{2} .
$$

But we know that $d f(a)$ and $\widehat{d^{2}} f(a)$ are absolutely 1-summing (see [4, Corollary $4.4(2)]$ ) and $\operatorname{id}_{E}$ is $(2 ; 1)$-summing (since $E$ is Hilbert) and hence we conclude that

$$
\sum_{j=j_{0}}^{\infty}\left\|f\left(a+x_{j}\right)-f(a)\right\|<\infty
$$

As an immediate consequence, we obtain:

Corollary 1. If $f$ is a holomorphic mapping defined on $A$ with values in $F$, then $f$ is Hilbert-Schmidt at $a \in A$ if, and only if, $f$ is absolutely 1-summing at $a$.

The same use of Taylor's formula gives us some variations of results of [4]:

TheOREM 5. Let $F$ be a Banach space, $E$ be a Banach space with the Orlicz property and $A$ an open set in $E$. If $f: A \subset E \rightarrow F$ is of class $C^{2}$ at $a \in A$ and $d f(a)$ is absolutely 1-summing, then $f$ is absolutely 1-summing at $a$.

Corollary 2. If $A$ is open in $l_{1}$ and $a \in A$, then every mapping $f$ : $A \rightarrow l_{2}$ of class $C^{2}$ at $a$ is absolutely 1-summing at a.

5.2. Hilbert-Schmidt vs absolutely $p$-summing mappings. We know that every continuous 2-homogeneous polynomial $P$ from $l_{2}$ into $\mathbb{K}$ is HilbertSchmidt at 0 , but we will show that if $1<p<\infty$, there is a continuous 2-homogeneous polynomial $P$ from $l_{2}$ into $\mathbb{K}$ that fails to be absolutely $p$-summing at zero.

We will consider the cases $p \geq 2$ and $1<p<2$. In both cases the polynomial is given by

$$
P\left(\left(\xi_{j}\right)_{j \in \mathbb{N}}\right)=\sum_{j \in \mathbb{N}} \xi_{j}^{2}
$$

CASE $p \geq 2$. We know that the natural orthonormal basis $\left(e_{j}\right)_{j \in \mathbb{N}}$ is in $l_{2}^{w}\left(l_{2}\right)$, hence in $l_{p}^{w}\left(l_{2}\right)$ for $p \geq 2$. Since $P\left(e_{j}\right)=1$ for all $j \in \mathbb{N}$, we cannot have $\left(P\left(e_{j}\right)\right)_{j \in \mathbb{N}} \in l_{p}$.

CASE $1<p<2$. Set $s=2 /(2-p)$. Then $1=1 / s+p / 2$ and $p s>2 p$. Now choose $\left(\lambda_{j}\right)_{j \in \mathbb{N}}$ in $l_{p s}$ but not in $l_{2 p}$. By the Hölder inequality involving $s$ and $2 / p$, we show that $\left(\lambda_{j} e_{j}\right)_{j \in \mathbb{N}} \in l_{p}^{w}\left(l_{2}\right)$. Since $P\left(\lambda_{j} e_{j}\right)=\lambda_{j}^{2}$ for each $j \in \mathbb{N}$, it follows that $\left(P\left(\lambda_{j} e_{j}\right)\right)_{j \in \mathbb{N}} \notin l_{p}$. 
Theorem 6. Let $0<p<\infty$. If $f$ defined on $A$ with values in $F$ is holomorphic and absolutely p-summing, then $f$ is Hilbert-Schmidt at a .

Proof. In this case we know that $(k !)^{-1} \widehat{d^{k}} f(a)$ is absolutely $p$-summing at 0 for each $k \in \mathbb{N}$, and there are $D \geq 0$ and $d>0$ satisfying

$$
\left\|\frac{1}{k !} \widehat{d}^{k} f(a)\right\|_{\mathrm{as}, p} \leq D d^{k} \quad \text { for every } k \in \mathbb{N} .
$$

If $\left(u_{i}\right)_{i \in I}$ is an orthonormal basis in $E$ and $J$ is a finite subset of $I$, we can write

$$
\sum_{j \in J}\left\|f\left(a+\lambda_{j} u_{j}\right)-f(a)\right\| \leq \sum_{j \in J} \sum_{k=1}^{\infty}\left\|\frac{1}{k !} \widehat{d}^{k} f(a)\left(\lambda_{j} u_{j}\right)\right\|=(*) .
$$

By Example 6 and the fact that a linear absolutely $p$-summing operator is Hilbert-Schmidt, we can write

$$
(*) \leq\|d f(a)\|_{\mathrm{HS}}\left\|\left(\lambda_{j}\right)_{j \in J}\right\|_{2}+\sum_{k=2}^{\infty}\left\|\frac{1}{k !} \widehat{d}^{k} f(a)\right\|\left\|\left(\lambda_{j}\right)_{j \in J}\right\|_{2}^{k}=(* *)
$$

for all $\left\|\left(\lambda_{j}\right)_{j \in J}\right\|_{2} \leq 1$. Since

$$
\left\|\frac{1}{k !} \widehat{d}^{k} f(a)\right\| \leq\left\|\frac{1}{k !} \widehat{d}^{k} f(a)\right\|_{\mathrm{as}, p} \leq D d^{k}
$$

for every natural $k$, we can write

$$
\begin{aligned}
(* *) & \leq\|d f(a)\|_{\mathrm{HS}}\left\|\left(\lambda_{j}\right)_{j \in J}\right\|_{2}+D d\left\|\left(\lambda_{j}\right)_{j \in J}\right\|_{2} \sum_{k=2}^{\infty} \frac{d^{k-1}}{(2 d)^{k-1}} \\
& =\left(\|d f(a)\|_{\mathrm{HS}}+D d\right)\left\|\left(\lambda_{j}\right)_{j \in J}\right\|_{2}
\end{aligned}
$$

if $\left\|\left(\lambda_{j}\right)_{j \in J}\right\|_{2} \leq \min \{1,1 / 2 d\}$. This proves our result.

REMARK 2. Our previous results show that the converse of Theorem 6 is not true for $p>1$.

5.3. Hilbert-Schmidt mappings of several variables. Following the idea of introducing the concept of absolute summability for arbitrary mappings of several variables, in this section we define Hilbert-Schmidt mappings of several variables and relate this concept to fully summing mappings.

Definition 5. Let $E_{1}, \ldots, E_{n}$ and $F$ be Hilbert spaces over $\mathbb{K}$, and $A \subset E_{1} \times \cdots \times E_{n}$ be an open set. A map $f: A \rightarrow F$ is Hilbert-Schmidt at $a=\left(a_{1}, \ldots, a_{n}\right) \in A$ if, for each orthonormal basis $\left(u_{i}^{(k)}\right)_{i \in I_{k}}$ in $E_{k}$, there exist $M \geq 0$ and $\delta>0$ so that

$$
B_{\delta}\left(a_{1}\right) \times \cdots \times B_{\delta}\left(a_{n}\right) \subset A
$$

and 


$$
\begin{aligned}
\sum_{j_{k} \in J_{k}, k=1, \ldots, n} \| f\left(a_{1}+\lambda_{j_{1}}^{(1)} u_{j_{1}}^{(1)}, \ldots,\right. & \left.a_{n}+\lambda_{j_{n}}^{(n)} u_{j_{n}}^{(n)}\right)-f\left(a_{1}, \ldots, a_{n}\right) \| \\
& \leq M\left\|\left(\lambda_{j}^{(1)}\right)_{j \in J_{1}}\right\|_{2} \cdots\left\|\left(\lambda_{j}^{(n)}\right)_{j \in J_{n}}\right\|_{2}
\end{aligned}
$$

for all finite subsets $J_{k} \subset I_{k}, \lambda_{j}^{(k)} \in \mathbb{K}$ with $\left\|\left(\lambda_{j}^{(k)}\right)_{j \in J_{k}}\right\|_{2}<\delta$.

Proposition 2. If $f: A \subset E_{1} \times \cdots \times E_{n} \rightarrow F$ is fully $(1 ; 1, \ldots, 1)$ summing at $a \in A$, then $f$ is Hilbert-Schmidt at $a$.

Proof. Analogous to the proof of Proposition 1.

ExAMPLE 7 . If $\varphi_{j}: E_{j} \rightarrow \mathbb{K}, j=1, \ldots, n-1$, are continuous linear functionals and $g: E_{n} \rightarrow F$ is absolutely 1 -summing at 0 , with $g(0)=0$, then $f: E_{1} \times \cdots \times E_{n} \rightarrow F$ given by $f\left(x_{1}, \ldots, x_{n}\right)=\varphi_{1}\left(x_{1}\right) \cdots \varphi_{n-1}\left(x_{n-1}\right) g\left(x_{n}\right)$ is Hilbert-Schmidt at 0 .

We thank the copy editor, Jerzy Trzeciak, for the improvements in the presentation of the paper, and the referee for several helpful comments.

\section{References}

[1] R. Alencar and M. C. Matos, Some classes of multilinear mappings between Banach spaces, Publ. Departamento Análisis Matematico, Univ. Complutense Madrid, Section 1, no. 12 (1989).

[2] F. Bombal, D. Pérez-García and I. Villanueva, Multilinear extensions of Grothendieck's theorem, Quart. J. Math. 55 (2004), 441-450.

[3] M. C. Matos, Absolutely summing holomorphic mappings, An. Acad. Bras. Ciênc. 68 (1996), 1-13.

[4] —, Nonlinear absolutely summing mappings, Math. Nachr. 258 (2003), 71-89.

[5] - Fully absolutely summing and Hilbert-Schmidt multilinear mappings, Collect. Math. 54 (2003), 111-136.

[6] D. M. Pellegrino, Almost summing mappings, Arch. Math. (Basel) 82 (2004), 68-80.

[7] D. M. Pellegrino and M. L. V. Souza, Fully summing multilinear and holomorphic mappings into Hilbert spaces, Math. Nachr. 278 (2005), 877-887.

[8] D. Pérez-García, The inclusion theorem for multiple summing operators, Studia Math. 165 (2004), 275-290.

[9] D. Pérez-García and I. Villanueva, Multiple summing operators on Banach spaces, J. Math. Anal. Appl. 285 (2003), 86-96.

[10] M. L. V. Souza, Aplicações multilineares completamente absolutamente somantes, Doctoral thesis, Universidade Estadual de Campinas, 2003.

\section{IMECC-UNICAMP}

Caixa Postal 6065

Campinas, SP, Brazil

E-mail: matos@ime.unicamp.br
Departamento de Matemática Universidade Federal da Paraíba Cep 58051-900 João Pessoa, PB, Brazil E-mail: dmpellegrino@gmail.com 